\title{
Data Aggregation and Life Time Improvement in wireless Sensor Networks using Dynamic Clustering
}

\author{
Reepu Daman \\ Research Scholar at \\ department of Computer \\ Science \&Engg. \\ Sachdeva Engg. College for \\ Girls, Gharuan
}

\author{
Preety Chaudhary \\ Assistant Professor at \\ department of Computer \\ Science \&Engg. \\ Sachdeva Engg. College for \\ Girls, Gharuan
}

\author{
Rakesh Kumar \\ Assistant Professor at \\ department of Computer \\ Science \&Engg. \\ Sachdeva Engg. College for \\ Girls, Gharuan
}

\begin{abstract}
Wireless sensor network is an emerging area of research due to vulnerability of sensing information from not approachable areas. In WSN sensor nodes have been deployed for sensing information and transmit this information to base station so that various decisions can be developed. In the processing of WSN data aggregation and energy consumption are major issues. In this paper a new approach has been purposed for data management and energy consumption reduction using dynamic clustering and avoidance ofredundant information transmission over the network. This approach use check sum approach for data redundancy checking and discard redundant or repeated information. This approach provides better results than previous approaches.
\end{abstract}

\section{Keywords}

Energy optimization, WSN, data sensing, clusters.

\section{INTRODUCTION}

Wireless Sensor Network took worldwide attention latently because of advances made wireless communication, information and electronics fields. Presently mostly wireless sensors devices are considerably constraint in term of computational constraints in term of computation power, memory, efficiency, computation power because of technology and reasons. Mostly Research on WSN's has focused on the design of energy and computational efficiency algorithms and protocols. WSN consist of number of sensor nodes. These have limited power with irreplaceable battery. Processor have some limitations and by considering the limitations of these sensor nodes. Not only the good communication is required but also the network life time should be long as much as possible in the applications of wireless sensor networks.

\subsection{Mac Protocols}

\section{- BMAC}

BMAC [2] is an asynchronous and light weight sensor network MAC Protocol. Like an asynchronous and light weight sensor network MAC protocol that aims at providing versatile medium access while keeping the MAC functionality as simple as possible. As an asynchronous protocol, BMAC eliminates the communication and processing overhead for scheduling and synchronization, which reduces energy consumption. BMAC enables each node to wake up periodically to check for channel activity. The wake-up period is referred to as the check interval. BMAC defines eight check intervals, and each check interval corresponds to one of BMAC's eight listening modes.

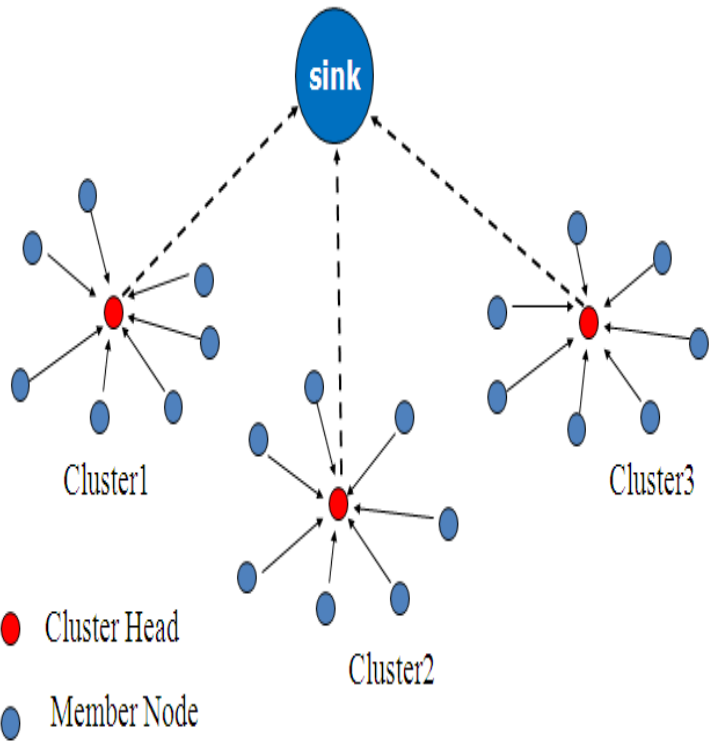

Fig 1WSN Clustering Architecture

To ensure that all packet are heard by neighboring nodes, packets are sent with a preamble whose receiving time is larger than the check interval. BMAC define eight different preamble length referred to as transmit nodes.

- DMAC

DMAC [2] is scheduled based MAC protocol which has been designed $\&$ optimized for tree. The main objective of this MAC protocol is to achieve low latency and still maintaining the energy efficiency. In this protocol the time is divided in small slots and runs carrier sensing multiple access (CSMA) [3] with acknowledgement within each slot to transmit/receive one packet. The sensor node periodically executes the basic sequence of ' 1 ' transmit, ' 1 ' receive and 'n' sleep slots.

\section{- $\quad$ RFID impulse}

RFID Impulse is a very low-power radio wakeup scheme for sensor networks that relies on off-the shelf RFID [4] readers and tags. The Basis functionality of RFID Impulse is that all network nodes turn off their radios, including the voltage regulator and the oscillator, as long as they have no packets to send or receive and sends a short acknowledgment message through the standard radio to the sender, indicating that the RFID wake-up was successful. Upon receiving the 
short acknowledgment from the receiver, the sender commences the transmission. Once the sender completes all its packet transmissions, both sender and receiver again turn off their radios If a sender fails to receive an acknowledgment from the If a sender fails to receive an acknowledgment from the receiver in response to an RFID wake-up signal, the sender assumes that the receiver tag did not detect the signal or that the signal level is too low to activate the receiver tag. The sender then transmits the RFID wake-up signal again, with a maximum of three retries. If the receiver acknowledges receipt of one of the RFID wake-up signals, then the sender proceeds with the transmission. Otherwise, the sender ceases its attempts to use RFID wakeup signals to this receiver.

\section{- $\quad$ Trade-Offs}

To address the energy trade-off, RFID Impulse supports traffic-based selection of low-power radio modes. As a general rule, when the traffic load is high in a particular region of the network, nodes use lighter sleep modes as they have to wake up frequently to send and receive packets. It is not worthwhile for nodes to go into deeper sleep modes due to the higher latency and switching energy involved in frequent wake-up transitions. When the traffic load is low in a particular region of the network, switching between sleep and active states is less frequent, so nodes use deeper sleep modes that provide the highest energy savings. Determining quantitative thresholds for optimal sleep mode selection demands an energy model that captures all components contributing to energy consumption at sensor nodes.

\section{RELATED WORK}

Abusaimeh [5] "Energy-aware optimization of the number of clusters and cluster-heads in WSN" Enhancing the system lifetime and sparing vitality are the execution estimation in planning any Wireless Sensor Network. Grouping the remote sensor hubs and picking pioneer hubs to total the sending information are viewed as a primary method for sparing vitality and expanding the system lifetime. Numerous analysts have taken a shot at outlining grouping conventions for the Wireless Sensor Network. Be that as it may, few of the bunching calculations have concentrated on the quantities of groups required in every system and the ideal bunch heads in every group. Little of these bunching calculations consider the vitality level of the remote sensor hubs to decide what number of groups are required in the system and which hub ought to go about as the ideal group leader of the bunch. In this paper, we have proposed another method to decide the quantity of bunches and pick the best hub to be the group heads in the Wireless Sensor Network in view of the vitality level of the remote sensor hubs. We have contrasted this system and the inherent bunch tree strategy in building up the system and connecting the hubs to each other in the most recent sensor standard "ZigBee". In view of the reproduction comes about, the proposed grouping procedure has expanded the lifetime of the remote sensor organize by half in normal contrasting and the first lifetime of the bunch tree arrange.

Bojan S. [6] "Genetic algorithm as energy optimization method in WSN" In this paper we exhibit a strategy for minimization of vitality utilization amid parcel sending technique in remote sensor systems using hereditary calculation. The proposed arrangement relies on upon cautious perception of the enhancement space and finish Customization of hereditary calculation to suit the particular sort of vitality capacity. Along these lines, vitality least can be found with more than $99.9 \%$ accuracy with extra minimization of blunder or memory space or CPU utilization, in the meantime, as appeared in this paper.

Jackulin, T. [7] "Energy optimization for WSN architecture and self-test Embedded processor" Internet testing of processors incorporated in WSN hubs works on battery, and battery span decide the lifetime of the framework, this paper proposes a new bunching Embedded frameworks less asset use. Grouping is another way to deal with effectively use the vitality of sensor hubs, this approach lessens the system activity and also assets use, we propose another vitality proficient and dependable bunching calculation called onscreen character coordinated bunching convention (ADCP) that expands the life time of system and our reproduction brings about productively convey information to a performer hub with least deferral, that aides for making a fast move and control the assault in its underlying stage. Performing artist Directed Clustering convention (ADCP) calculation is utilized for decision and keeping up system movement bunching Technique is changed for WSN, Low power proportion can be accomplished. A connection life time expectation directing calculation can be included with the ADCP convention. Recreation comes about demonstrate that vitality investment funds at processor level are up to $40 \%$ percent.

Tanevski, P. [8] "Power supply energy optimization for ultra-low-power wireless sensor nodes" The remote sensor hubs are most vitality productive when they work at the negligible working voltage. The ostensible voltage of the batteries utilized is frequently altogether higher. This paper examines DC-DC control change from the perspective of ultra-low-control remote sensor hubs. Utilizing an altered circuit of an appropriate business DC-DC converter and a super capacitor, this paper demonstrates that it is conceivable to amplify battery lifetime of a remote sensor hub for certain range of uses. The proficiency of the DC-DC change and also super capacitor charge-release effectiveness have been examined and measured. The impact of battery release streams and spillage ebbs and flows is additionally introduced. Moreover, in this paper, we diagram the particulars of a module required between the battery and the sensor hub that will be controlled by the hub's MCU and will keep the hub working at insignificant operational voltage while in the meantime expanding the vitality conveyed from the battery to the hub. Therefore, this paper likewise goes for setting establishments for future advancement of a vitality enhancement module for ultra-low power remote sensor hubs.

\section{PROPOSED WORK}

WSN has been used for sensing information from a particular location in which human interaction is not possible. Sensor nodes deployed in the network has been used for sensing information and transmitting sensed information to base station for decision evaluating process. Sensor nodes transmit sensor information to base station with utilization of intermediate nodes for data transmission. In the purposed work data has been transmitted with the help of intermediate nodes to base station.

In the purposed work dynamic clustering has been used for transmitting data to sink nodes that avoid congestion and data aggregation has been done to an extent approach.

In the purposed work network has been divided into different clusters that has been used for cluster head selection. In this process clusters have been divided on the basis of nodes properties. These nodes have been divided into different 
clusters and cluster head selection has been done on the basis of maximum energy containing node.

In the process of dynamic clustering in WSN two different phases have been taken place. These phases are steady phase and cluster head selection phase. In the process of cluster head selection nodes cluster head probability and nodes energy has been utilized.

\section{- Cluster Head Selection}

In the process of cluster head selection network has been divided into different clusters that have been used for cluster head selection phase. In this process energy of each node has been measured that has been used for selection of cluster head after each round.

$$
\begin{aligned}
& T(n)=\frac{P * E_{n \_ \text {Energy }}}{1-P *\left(r * \bmod P^{-1}\right) * E_{\text {Intial }}} \\
& T(n)=0
\end{aligned}
$$

In equation (1) $\mathrm{P}$ denotes probability of a node to be cluster head and $\mathrm{n}$ is a random number between 0 and 1 . $E_{n \_ \text {Energy }}$ Represents energy contains a particular node at selection time and $E_{\text {Intial }}$ denotes maximum amount of energy having a node at simulation setup time.

On the basis of these values cluster head for a cluster has been selected that has been used for cluster head selection. This phase is reoccurred after each round that has been executed. In this process different nodes have a chance to become a cluster head.

Cluster in a cluster is utilized to receives data from other cluster members and transmit this information to other sink nodes directly or by using other cluster heads.

\section{- Data Aggregation}

After selection of cluster head all the nodes available in a single cluster start transmitting sensed information to cluster head. Cluster head collects all the information and transmit information to base station. In this process node consumes energy that is data sensing, data transmitting, data receiving and data aggregation energy. To optimize energy consumed in data aggregation process clustering node has been used for check redundancy in the gathered information. To check redundant checksum approach has been used that use sum of the bits has been stored in a packet and at receiving end check sum bit has been matched with previous collected information. On the basis of checksum bit data collected has verified. At this phase redundant information has been dropped out and the amount of energy that has been used reduced and less amount of data have to be transmitted over the network that increase lifetime of the network.

\section{- Data Transmission using Routing Protocol}

Sensing nodes available in the network transmit sensing information to cluster head or sub cluster head using routing protocol routes. In the purposed work dynamic route selection approach has been used for transmission of information from single node to cluster head or sub cluster head. Sensor nodes can communicate directly with cluster head or utilized intermediate nodes for transmission of information. After receiving information from nodes sub cluster head communicate with cluster head and cluster head will transmit data directly to base station or to other cluster head that are nearby to base station.

\section{RESULTS AND DISCUSSIONS}

Data aggregation in WSN has been done to optimize energy consumption in WSN so that easily data can be transmitted over the network and network lifetime gets increases. In this process WSN has been setup using NS-2 Simulator. Wireless sensor network utilize different simulation parameters for initialization and sensing information. Sensing information has been transmitted to base station for decision making process. In this proposed work data aggregation has been done in such a way so that minimum redundancy occurred over the cluster head and network overhead get decrease and lifetime gets increase.

In the purposed work simulation parameters have been initialized for WSN. These parameters are described below.

Table 4.1 Simulation parameters Setup

\begin{tabular}{|c|c|}
\hline Parameter & Description \\
\hline Area & $1500 * 1500$ \\
\hline Number of nodes & Omni \\
\hline Antenna & Drop Tail \\
\hline Queue Type & 250 \\
\hline Queue Length & LEACH \\
\hline Routing Protocol & $8.02 / 11$ \\
\hline MAC Type & $0.5 \mathrm{~J}$ \\
\hline Receiving Energy & $0.9 \mathrm{~J}$ \\
\hline Transmission Energy & 100 Jules \\
\hline Initial Energy & ENERGY Model \\
\hline Energy Model & \\
\hline
\end{tabular}

Table 4.1 defines various simulation parameters that are necessary for simulation of the purposed work. In the process on WSN these parameters are utilized for initialization of the simulation setup. In the WSN 55 nodes have been deployed in the network that have been used for sensing information and transmitting information to $50^{\text {th }}$ node that is act as a base station from the purposed work. In the purposed work various parameter have been analyzed for performance evaluation of purposed work. These parameters are illustrated below.

\section{- $\quad$ Packet Delivery Ratio}

It is defined as the ratio between total numbers of packets delivered properly to destination to total number of packets generated by sources.

$$
P D R=\frac{P_{d}}{P_{S}}(3)
$$

- $\quad$ Throughput

It is defined as the average number of bytes has been delivered in a particular unit of time. Throughput of the system has been measured by ratio of product packet size in bytes total packet delivered tototal simulation time. 


$$
\text { Throghput }=\frac{P_{d} * S}{T}(4)
$$

- Network Lifetime

It is defined as the total time for which network nodes have been awake and performing some actions. It is measured as the time duration from which simulation has been started to last node get exhaust its battery life and stop performing any action

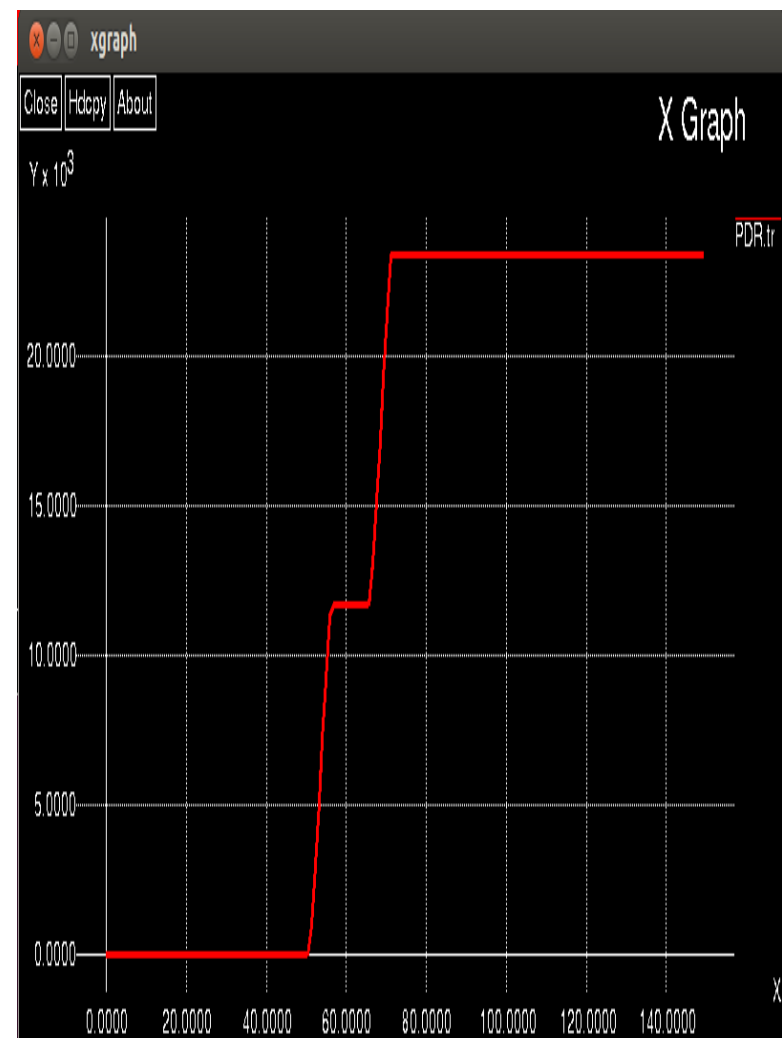

Fig 4.1 Packet Delivery Ratio

This figure represents packet delivery ratio of WSN. This figure represents number of packets that have been transmitted successfully over the network.

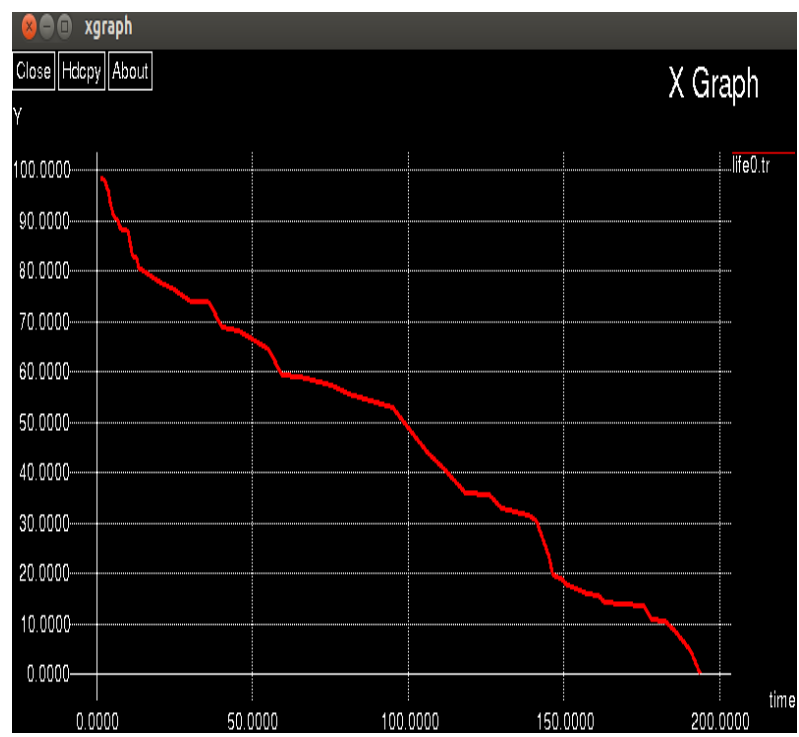

Fig 5.7 Life time
This figure is use to represent the Lifetime of a node. Lifetime is defined as the total time in which node can survive without any disturbance.

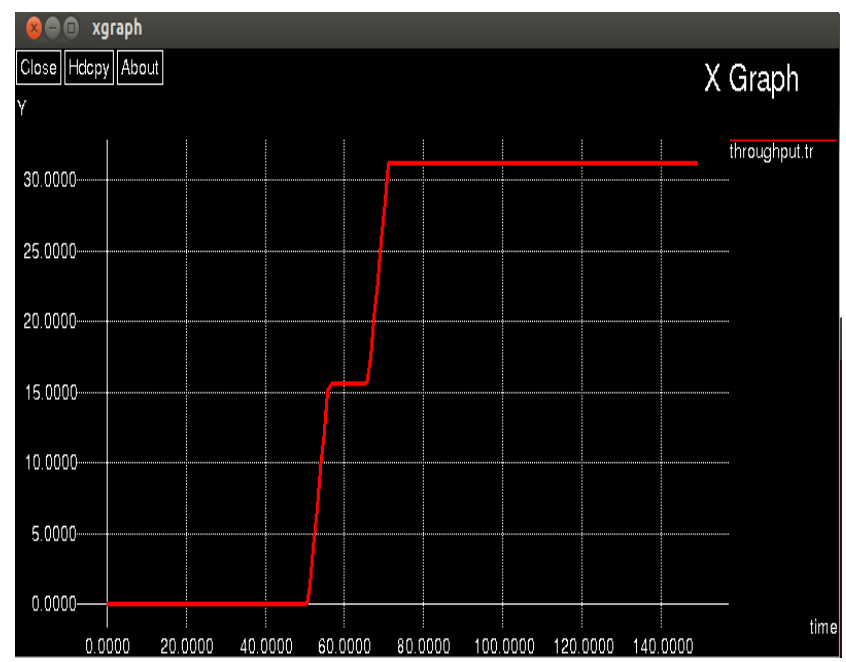

Fig 5.8 Throughput

This figure is use to represent the Throughput. Throughput is defined as the number bytes delivered successfully per unit interval of time over the network.

\section{CONCLUSION}

WSN is area of networking that deals with sensing and transmitting information to base station for decision making process. Various approaches had been purposed for data transmission over the network. These approaches consumes large amount of energy due to availability of redundant information at the receiving nodes. This cause various issue inperformances of network.In this paper a new approach has been purposed that works for data aggregation WSN using Dynamic clustering hybrid with checksum approach to avoid redundant information. By analyzing simulation parameters one can conclude that purposed approach provide better performance than previous approaches.

\section{REFERENCES}

[1] QiulingTang; Changyin Sun; Huan Wen; Ye Liang "Cross-layer energy efficiency analysis and optimization in WSN"IEEE conference on Networking, Sensing and Control (ICNSC), 2010, pp. $138-142$.

[2] Sharawi, M.; Emary, E.; Saroit, I.A.; El-Mahdy, H. "WSN's energy-aware coverage preserving optimization model based on multi-objective bat algorithm" IEEE conference on Evolutionary Computation (CEC), 2015 , pp. $472-479$.

[3] Darif, A.; Aboutajdine, D.; Saadane, R. "Energy consumption optimization in real time applications for WSN using IR-UWB technology" IEEE conference on Renewable and Sustainable Energy Conference (IRSEC), 2013, pp. 379 - 384.

[4] Ganhão, F. Pereira, M.; Bernardo, L.; Dinis, R. "Energy per Useful Packet Optimization on a TDMA WSN Channel" IEEE conference on Computer Communications and Networks (ICCCN), 2010, pp. 1 6.

[5] Abusaimeh, H.; Shuang-Hua Yang "Energy-aware optimization of the number of clusters and cluster-heads 
in WSN" IEEE conference on Innovations in Information Technology (IIT), 2012, pp. $178-183$.

[6] Bojan, S.; Nikola, Z. "Genetic algorithm as energy optimization method in WSN" IEEE conference on Telecommunications Forum (TELFOR), 2013, pp. 97 100.

[7] Jackulin, T.; Ramya, M.; Subashini, C. "Energy optimization for WSN architecture and self-test Embedded processor" IEEE conference on Emerging Trends in Electrical Engineering and Energy Management (ICETEEEM), 2012, pp. 253 - 256.

[8] Tanevski, M.; Boegli, A.; Farine, P. "Power supply energy optimization for ultra-low-power wireless sensor nodes" IEEE conference on Sensors Applications Symposium (SAS), 2013, pp.176 - 181 .

[9] Elshaikh, M. ; Bin MohdWarip, M.N. ; Lynn, O.B. ; Ahmad, R.B. "Energy consumption optimization with Ichi Taguchi method for Wireless Sensor Networks" IEEE conference on Electronic Design (ICED),2014,pp $493-498$

[10] Elhabyan, R. S.; Yagoub, M.C.E. "Particle swarm optimization protocol for clustering in wireless sensor networks: A realistic approach" IEEE conference on Information Reuse and Integration (IRI), 2014, pp. 345 -350 . 